\title{
ON THE TRAPEZOID QUADRATURE FORMULA FOR LIPSCHITZIAN MAPPINGS AND APPIICATIONS
}

\author{
SEVER SILVESTRU DRAGOMIR
}

\begin{abstract}
The estimation of the remainder term in trapezoid formula for lipschitzian mappings are given. Applications for special means are also pointed out.
\end{abstract}

\section{Introduction}

The following ineqality is well known in the literature as the trapezoid inequality:

$$
\left|\int_{a}^{b} f(x) d x-\frac{f(a)+f(b)}{2}(b-a)\right| \leq \frac{1}{12}\left\|f^{\prime \prime}\right\|_{\infty}(b-a)^{3}
$$

where the mapping $f:[a, b] \rightarrow R$ is supposed to be twice differentiable on the interval $(a, b)$ and having the second derivative bounded on $(a, b)$, that is $\left\|f^{\prime \prime}\right\|_{\infty}:=$ $\sup _{x \in(a, b)}\left|f^{\prime \prime}(x)\right|<\infty$.

Now, if we assume that $I_{h}: a=x_{0}<x_{1}<\cdots<x_{n-1}<x_{n}=b$ is a partition of the interval $[a, b]$ and $f$ is as above, then we have the trapezoid quadrature formula:

$$
\int_{a}^{b} f(x) d x=A_{T}\left(f, I_{h}\right)+R_{T}\left(f, I_{h}\right)
$$

where $A_{T}\left(f, I_{h}\right)$ is the trapezoid rule

$$
A_{T}\left(f, I_{h}\right)=: \frac{1}{2} \sum_{i=0}^{n-1}\left[f\left(x_{i}\right)+f\left(x_{i+1}\right)\right] h_{i}
$$

and the remainder term $R_{T}\left(f, I_{h}\right)$ satisfies the estimation

$$
\left|R_{T}\left(f, I_{h}\right)\right| \leq \frac{1}{12}\left\|f^{\prime \prime}\right\|_{\infty} \sum_{i=0}^{n-1} h_{i}^{3}
$$

where $h_{i}:=x_{i+1}-x_{i}$ for $i=0, \ldots, n-1$.

Received August 20, 1998.

1991 Mathematics Subject Classification. 26D15, 26D99.

Key words and phrases. Trapezoid inequality, special means. 
When we have an equidistant partitioning of $[a, b]$ given by

$$
I_{n}: x_{i}:=a+\frac{b-a}{n} i, \quad i=0, \ldots, n
$$

then we have the formula

$$
\int_{a}^{b} f(x) d x=A_{T, n}(f)+R_{T, n}(f)
$$

where

$$
A_{T, n}(f):=\frac{b-a}{2 n} \sum_{i=0}^{n-1}\left[f\left(a+\frac{b-a}{n} i\right)+f\left(a+\frac{b-a}{n}(i+1)\right)\right]
$$

and the remainder satisfies the estimation

$$
\left|R_{T, n}(f)\right| \leq \frac{1}{12} \cdot \frac{(b-a)^{3}}{n^{2}}\left\|f^{\prime \prime}\right\|_{\infty} .
$$

For other trapezoid type's inequalities see the recent book [1].

\section{Trapezoid Inequality for Lipschitzian Mappings}

The following trapezoid inequality for lipschitzian mappings holds: Theorem 2.1. Let $f:[a, b] \rightarrow R$ be an L-lipschitzian mapping on $[a, b]$. Then we
have the inequality

$$
\left|\int_{a}^{b} f(x) d x-\frac{f(a)+f(b)}{2}(b-a)\right| \leq \frac{1}{4} L(b-a)^{2} .
$$

The constant $\frac{1}{4}$ is the best possible one.

Proof. Using the integration by parts formula for Riemann-Stieltjes integral we have

$$
\int_{a}^{b}\left(x-\frac{a+b}{2}\right) d f(x)=\frac{f(a)+f(b)}{2}(b-a)-\int_{a}^{b} f(x) d x .
$$
If $p:[a, b] \rightarrow R$ is Riemann integrable on $[a, b]$ and $v:[a, b] \rightarrow R$ is $L$-lipschitzian on
$[a, b]$, then

$$
\left|\int_{a}^{b} p(x) d v(x)\right| \leq L \int_{a}^{b}|p(x)| d x .
$$

Applying the inequality (2.3) for $p(x)=x-\frac{a+b}{2}, v(x)=f(x), x \in[a, b]$, we get

$$
\left|\int_{a}^{b}\left(x-\frac{a+b}{2}\right) d f(x)\right| \leq L \int_{a}^{b}\left|x-\frac{a+b}{2}\right| d x .
$$


But

$$
\int_{a}^{b}\left|x-\frac{a+b}{2}\right| d x=\frac{(b-a)^{2}}{4}
$$

and then by (2.4), via the identity (2.2), we deduce the desired inequality (2.1).

Now, assume that the inequaltiy (2.1) holds with a constant $C>0$, i.e.,

$$
\left|\int_{a}^{b} f(x) d x-\frac{f(a)+f(b)}{2}(b-a)\right| \leq C L(b-a)^{2} .
$$

Consider the mapping $f:[a, b] \rightarrow R, f(x)=\left|x-\frac{a+b}{2}\right|$. Then

$$
|f(x)-f(y)|=|| x-\frac{a+b}{2}|-| y-\frac{a+b}{2} \| \leq|x-y|
$$

for all $x, y \in[a, b]$; which shows that $f$ is $L$-lipschitzian with the constant $L=1$.

For this mapping we have

$$
\int_{a}^{b} f(x) d x-\frac{f(a)+f(b)}{2}(b-a)=\frac{(b-a)^{2}}{4}
$$

and

and then by (2.5) we get

$$
L(b-a)^{2}=(b-a)^{2}
$$

$$
\frac{(b-a)^{2}}{4} \leq C(b-a)^{2}
$$

which implies that $C \geq \frac{1}{4}$ and the sharpness of (2.1) is proved.

The following corollary holds:

Corollary 2.2. Let $f:[a, b] \rightarrow R$ be a differentiable mapping on $(a, b)$ whose derivative is bounded on $(a, b)$. Then we have the inequality:

$$
\left|\int_{a}^{b} f(x) d x-\frac{f(a)+f(b)}{2}(b-a)\right| \leq \frac{1}{4}\left\|f^{\prime}\right\|_{\infty}(b-a)^{2} .
$$

Remark 2.3. It is well known that if $f:[a, b] \rightarrow R$ is a convex mapping on $[a, b]$, then Hermite-Hadamard's inequality holds

$$
\frac{f(a)+f(b)}{2}(b-a) \geq \int_{a}^{b} f(x) d x \geq f\left(\frac{a+b}{2}\right)(b-a) .
$$

Now, if we assume that $f: I \subset R \rightarrow R$ is convex on $I$ and $a, b \in \operatorname{Int}(I), a<b$; then $f_{+}^{\prime}$ is monotonous nondecreasing on $[a, b]$ and by Theorem 2.1 . we get

$$
0 \leq \frac{f(a)+f(b)}{2}-\frac{1}{b-a} \int_{a}^{b} f(x) d x \leq \frac{1}{4} \max \left\{\left|f_{+}^{\prime}(a)\right|,\left|f_{-}^{\prime}(b)\right|\right\}(b-a)
$$


which gives a counterpart for the second membership of Hadamard's inequality.

The following corollary for trapezoid composite formula holds:

Corollary 2.4. Let $f:[a, b] \rightarrow R$ be an L-lipschitzian mapping on $[a, b]$ and $I_{h} a$ partition of $[a, b]$. Then we have the trapezoid quadrature formula (1.2) and the remainder term $R_{T}\left(f, \dot{I}_{h}\right)$ satisfies the estimation:

$$
\left|R_{T}\left(f, I_{h}\right)\right| \leq \frac{1}{4} L \sum_{i=0}^{n-1} h_{i}^{2}
$$

Moreover, the constant $\frac{1}{4}$ is the best possible one.

The case of equidistant partitioning is embodied in the following corollary:

Corollary 2.5. Let $I_{n}$ be an equidistant partitioning of $[a, b]$ and $f$ be as in Theorem 2.1. Then we have the formula (1.6) and the remainder astisfies the estimation:

$$
\left|R_{T, n}(f)\right| \leq \frac{1}{4} \cdot \frac{L}{n}(b-a)^{2} .
$$

Remark 2.6. If we want to approximate the integral $\int_{a}^{b} f(x) d x$ by trapeziod formula $A_{T, n}(f)$ with an accuracy less that $\varepsilon>0$, we need at least $n_{\varepsilon} \in N$ points for the division $I_{n}$, where

$$
n_{\varepsilon}:=\left[\frac{1}{4} \cdot \frac{L}{\varepsilon}(b-a)^{2}\right]+1 .
$$

Comments 2.7. If the mapping $f:[a, b] \rightarrow R$ is neither twice differentiable nor the second derivative is bounded on $(a, b)$, then we can not apply the classical estimation in trapezoid formula using the second derivative. But if we assume that $f$ is lipschitzian, then we can use instead the formula (2.9).

We give have here a class of mappings which are lipschitzian but having the second derivative unbounded on the given interval.

Let $f_{p, q}:[a, b] \rightarrow R, f_{p, q}(x):=\left(x^{q}-a^{q}\right)^{p}$ where $p \in(1,2)$ and $q \geq 2$. Then obviously

and

$$
f_{p, q}^{\prime}(x):=p q x^{q-1}\left(x^{q}-a^{q}\right)^{p-1}, \quad x \in(a, b)
$$

$$
f_{p, q}^{\prime \prime}(x)=p q \frac{x^{q-2}\left[(p q-1) x^{q}-(q-1) a^{q}\right]}{\left(x^{q}-a^{q}\right)^{2-p}}, \quad x \in(a, b) .
$$

It is clear that $f$ is lipschitzian with the constant

but $\lim _{x \rightarrow a+} f_{p, q}^{\prime \prime}(x)=+\infty$.

$$
L:=p a b^{q-1}\left(b^{q}-a^{q}\right)^{p-1}<\infty
$$

\section{Applications for Special Means}

Let us recall the following means: 
1. Arithmetic mean

$$
A=A(a, b):=\frac{a+b}{2}, \quad a, b \geq 0
$$

2. Geometric mean

$$
G=G(a, b):=\sqrt{a b}, \quad a, b \geq 0
$$

3. Harmonic mean

$$
H=H(a, b):=\frac{2}{\frac{1}{a}+\frac{1}{b}}, \quad a, b>0
$$

4. Logarithmic mean

$$
L=L(a, b):=\frac{b-a}{\ln b-\ln a}, \quad a, b>0, a \neq b
$$

5. Identric mean

$$
I=I(a, b):=\frac{1}{e}\left(\frac{b^{b}}{a^{a}}\right)^{\frac{1}{b-a}}, \quad a, b>0, a \neq b
$$

6. p-Logarithmic mean

$$
L_{p}=L_{p}(a, b):=\left[\frac{b^{p+1}-a^{p+1}}{(p+1)(b-a)}\right]^{\frac{1}{p}}, \quad p \in R \backslash\{-1,0\}, \quad a, b>0, a \neq b .
$$

It is well known that $L_{p}$ is monotonous nondecreasing over $p \in R$ with $L_{-1}:=L$ and $L_{0}:=I$. In particular, we have the following inequalities

$$
H \leq G \leq L \leq I \leq A
$$

Now, using Theroem 2.1 we can also state the following inequalities:

1. Let $f:[a, b] \rightarrow R(0<a<b), f(x)=x^{p}, p \in R \backslash\{-1,0\}$. Then

$$
\left\|f^{\prime}\right\|_{\infty}=\delta_{p}(a, b):=\left\{\begin{array}{c}
p b^{p-1} \text { if } p \geq 1 \\
|p| a^{p-1} \text { if } p \in(-\infty, 1) \backslash\{-1,0\}
\end{array}\right.
$$

Using the inequality (2.6) we get

$$
\left|L_{p}^{p}(a, b)-A\left(a^{p}, b^{p}\right)\right| \leq \frac{1}{4} \delta_{p}(a, b)(b-a) .
$$

2. Let $f:[a, b] \rightarrow R(0<a<b), f(x)=\frac{1}{x}$. Then

$$
\left\|f^{\prime}\right\|_{\infty}=\frac{1}{a^{2}}
$$

Using the inequality (2.6) we get

$$
0 \leq L-H \leq \frac{b-a}{4 a^{2}} L H
$$


3. Let $f:[a, b] \rightarrow R(0<a<b), f(x)=\ln x$. Then

$$
\left\|f^{\prime}\right\|_{\infty}=\frac{1}{a} .
$$

Using the inequality (2.6) we get

$$
1 \leq \frac{I}{G} \leq \exp \left(\frac{b-a}{4 a}\right) .
$$

\section{References} [1] D. S. Mitrinović, J. E. Pečarić and A. M. Fink, Inequalities for Functions and their Integrals
and Derivatives, Kluwer Academic Publishers, 1994.

Department of Computer and Mathematical Sciences, Victoria University of Technology, P. O. Box 14428, Melbourne City, MC 8001, Victoria, Australia.

E-mail: sever@matilda.vu.edu.au 